Arq. Bras. Med. Vet. Zootec., v.70, n.2, p.511-516, 2018

\title{
Osteossíntese de fêmur e tíbia em veado-catingueiro (Mazama gouazoubira): relato de caso
}

\author{
[Osteosynthesis of the femur and tibia of a brown brocket deer \\ (Mazama gouazoubira): case report] \\ L.E. Surita, B. Zafalon-Silva, E.A.R. Santos, M.M. Alievi* \\ Universidade Federal do Rio Grande do Sul - UFRGS - Porto Alegre, RS \\ RESUMO
}

\begin{abstract}
O presente estudo relata o caso de um veado-catingueiro jovem, provindo de vida livre e com histórico de atropelamento. Posteriormente à realização de exames, o animal foi diagnosticado com fratura exposta distal de tíbia (Salter-Harris tipo I) e fratura cominutiva de metáfise distal de fêmur, ambas em membro pélvico esquerdo. Para a osteossíntese da tíbia, foram utilizados pinos intramedulares de Steinmann inseridos pelos maléolos lateral e medial, de forma cruzada. A osteossíntese do fêmur foi realizada com associação de dois pinos de Steinmann, conforme técnica modificada de Rush, e placa de compressão dinâmica. As técnicas cirúrgicas adotadas foram satisfatórias para estabilização, consolidação de ambas as fraturas e retorno funcional do membro. Após 90 dias da cirurgia, o animal foi reintroduzido à natureza.
\end{abstract}

Palavras-chave: cervidae, ortopedia, fratura distal

\begin{abstract}
The present study reports the case of a juvenile wild brown brocket deer, with car accident history. After a complete examination, the animal was diagnosed with open distal tibial fracture (Salter-Harris type I) and comminuted fracture of the distal metaphysis of the femur, both in the left hindlimb. For the tibial osteosynthesis, Steinmann pins were used intramedullary, inserted by the lateral and medial malleolus, crosswise. The osteosynthesis of the femur was performed with a combination of two Steinmann pins, as modified Rush technique, and a dynamic compression plate. The surgical techniques used were satisfactory for stabilization, consolidation of both fractures and function return of the member. Ninety days after surgery the animal was reintroduced to nature.
\end{abstract}

Keywords: cervidae, orthopedics, distal fracture

\section{INTRODUÇÃO}

O veado-catingueiro (Mazama gouazoubira) é uma espécie de cervídeo de pequeno porte que possui alta plasticidade fenotípica, adaptado a diversos ambientes com elevada interferência antrópica, sendo considerada uma espécie vulnerável e em perigo em alguns estados do país (Duarte e Reis, 2012). O atendimento desses animais em centros de triagem, criadouros e zoológicos é frequente devido a acidentes e traumas por interação antrópica (Duarte e Reis,

Recebido em 23 de novembro de 2016

Aceito em 24 de maio de 2017

* Autor para correspondência (corresponding author)

E-mail: marcelo.alievi@ufrgs.br
2012). As fraturas estão entre as lesões mais comuns, geralmente ocasionadas por traumas, o que demanda muitos estudos referentes às suas formas de tratamento (Nisbet et al., 2010).

As técnicas de reparação de fraturas em cervídeos citadas na literatura não diferem daquelas utilizadas em outras espécies domésticas. Fatores como idade, tamanho do animal, comportamento, procedência, gravidade e localização da fratura são determinantes para a escolha das diferentes abordagens terapêuticas (Black-Décima et al., 2010). 
Partindo desse contexto, o presente trabalho tem como objetivo relatar um caso de osteossíntese de fratura distal exposta de tíbia associada à fratura distal de fêmur em veado-catingueiro (Mazama gouazoubira) provindo de vida livre.

\section{CASUÍSTICA}

Um veado-catingueiro, fêmea, jovem, com 15,3 quilogramas de massa corporal, proveniente de vida livre, foi encaminhado para atendimento hospitalar com histórico de atropelamento. $\mathrm{Na}$ inspeção, foi observada claudicação grau 4, associada à fratura distal exposta tipo II da tíbia esquerda. Para realização do exame físico e imobilização do membro, foi efetuada contenção química utilizando cloridrato de cetamina $(10 \mathrm{mg} / \mathrm{kg}$, i.m.) e midazolam $(0,5 \mathrm{mg} / \mathrm{kg}$, i.m.) Posteriormente ao exame clínico geral do animal, foi realizada a limpeza da ferida com solução de $\mathrm{NaCl} 0,9 \%$, redução manual da fratura de tíbia e imobilização da região com bandagem de Robert Jones modificada. Ainda no exame, foi observado aumento de volume e hematoma na região do fêmur ipsilateral.

Em exame radiográfico, foi diagnosticada fratura distal de tíbia (Salter-Harris tipo I) e fratura cominutiva de metáfise distal de fêmur, ambas em membro pélvico esquerdo (Fig. 1). Como terapêutica, foi instituído antibiótico com associação de penicilinas e estreptomicinas
$(0,1 \mathrm{~mL} / \mathrm{kg}$, i.m., q.48h, cinco dias $)$, meloxicam $(0,2 \mathrm{mg} / \mathrm{kg}$, i.m., sid, três dias) e cloridrato de tramadol $(5 \mathrm{mg} / \mathrm{kg}$, i.m, sid, um dia). Após realização de exames hematológicos, o animal foi encaminhado para realização do procedimento cirúrgico 72 horas após o atendimento.

A medicação pré-anestésica foi cetamina (10mg/kg, i.m.) e midazolam $(0,5 \mathrm{mg} / \mathrm{kg}$, i.m.). Após tricotomia em toda extensão do membro afetado e da região lombar, utilizou-se propofol $(5 \mathrm{mg} / \mathrm{kg}$, i.v.) para indução anestésica. A intubação orotraqueal foi realizada com traqueotubo 6,5 com balonete, e a manutenção do plano anestésico foi obtida com isoflurano vaporizado com oxigênio a $100 \%$ em sistema circular com reinalação de gases. A antissepsia das regiões de acesso cirúrgico foi realizada com clorexidina 2\%. Para o bloqueio peridural, administrou-se sulfato de morfina $1 \%(0,1 \mathrm{mg} / \mathrm{kg})$ e cloridrato de bupivacaína $0,5 \%$ sem vasoconstritor $(1 \mathrm{mg} / \mathrm{kg})$. A profilaxia antimicrobiana foi realizada com cefalotina (30mg/kg, i.v.), e a fluidoterapia com solução de ringer lactato de sódio $(10 \mathrm{~mL} / \mathrm{kg} / \mathrm{h}$, i.v. $)$. Parâmetros anestésicos, como oximetria de pulso, eletrocardiografia (ritmo e traçado), temperatura esofágica e pressão arterial não invasiva, foram aferidos durante o procedimento (Tab. 1).

Tabela 1. Parâmetros anestésicos aferidos durante anestesia inalatória em um veado-catingueiro (Mazama gouazoubira) para realização de osteossíntese de fêmur e tíbia

\begin{tabular}{|ccccccccccc|}
\hline Tempo (min) & 0 & 10 & 20 & 30 & 40 & 50 & 60 & 70 & 80 & 90 \\
\hline FC (bpm) & 108 & 107 & 100 & 95 & 93 & 99 & 101 & 96 & 100 & 106 \\
FR (mpm) & 22 & 24 & 24 & 21 & 27 & 19 & 23 & 16 & 12 & 17 \\
SpO2 (\%) & 99 & 98 & 97 & 99 & 98 & 99 & 99 & 98 & 98 & 98 \\
PAS (mmHg) & 130 & 128 & 124 & 126 & 122 & 127 & 116 & 121 & 115 & 124 \\
PAM $(\mathrm{mmHg})$ & 95 & 98 & 90 & 102 & 101 & 85 & 81 & 72 & 69 & 76 \\
PAD (mmHg) & 60 & 72 & 54 & 69 & 69 & 66 & 49 & 33 & 29 & 51 \\
T $\left({ }^{\circ} \mathrm{C}\right)$ & 37 & 36,8 & 36,7 & 36,6 & 36,6 & 36,6 & 36,6 & 36,4 & 36,4 & 36,6 \\
\hline
\end{tabular}

$\mathrm{FC}=$ frequência cardíaca, $\mathrm{FR}=$ frequência respiratória, $\mathrm{SpO} 2=$ saturação de oxigênio, $\mathrm{PAS}=$ pressão arterial sistólica, $\mathrm{PAM}=$ pressão arterial média, $\mathrm{PAD}=$ pressão arterial diastólica, $\mathrm{T}\left({ }^{\circ} \mathrm{C}\right)=$ temperatura esofágica

O foco de fratura da tíbia foi acessado pela própria lesão cutânea na face medial. A fratura foi reduzida e, após, foi estabilizada pela inserção de quatro pinos de Steinmann, de maneira normógrada, através do maléolo lateral e medial, em direção à cortical oposta do segmento proximal. A dermorrafia foi realizada com fio não absorvível monofilamentar 2-0 em padrão interrompido simples.

A osteossíntese do fêmur foi realizada por acesso lateral e artrotomia do joelho. A fratura foi reduzida e, após, foi estabilizada com a introdução de dois pinos de Steinmann 3,5mm, conforme técnica modificada de Rush. Uma 
placa de compressão dinâmica de baixo contato (LC-DCP) de 3,5mm foi moldada e adaptada à superfície femoral lateral e fixada com parafusos de $3,5 \mathrm{~mm}$, sendo quatro proximais e dois distais ao foco da fratura. A artrorrafia e a síntese da fáscia lata foram realizadas com fio não absorvível monofilamentar 0 em padrão sultan. O tecido subcutâneo e a pele foram aproximados com fio não absorvível monofilamentar 2-0 em padrão contínuo simples e interrompido simples, respectivamente. A ferida cirúrgica foi mantida com curativo.
O exame radiográfico no pós-operatório imediato revelou que as fraturas apresentavam-se alinhadas e coaptadas (Fig. 1). Como medicações pós-operatórias, foram utilizados meloxicam $(0,2 \mathrm{mg} / \mathrm{kg}$, i.m., sid, cinco dias) e antibiótico com associação de penicilinas e estreptomicinas $(0,1 \mathrm{~mL} / \mathrm{kg}$, i.m., q. $48 \mathrm{~h}$, cinco dias $)$. A troca do curativo e a limpeza da ferida cirúrgica foram realizadas a cada sete dias. $\mathrm{O}$ animal foi mantido em uma baia, com dimensões de $12 \mathrm{~m}^{2}$, com mínima exposição ao contato humano durante todo o período da internação.

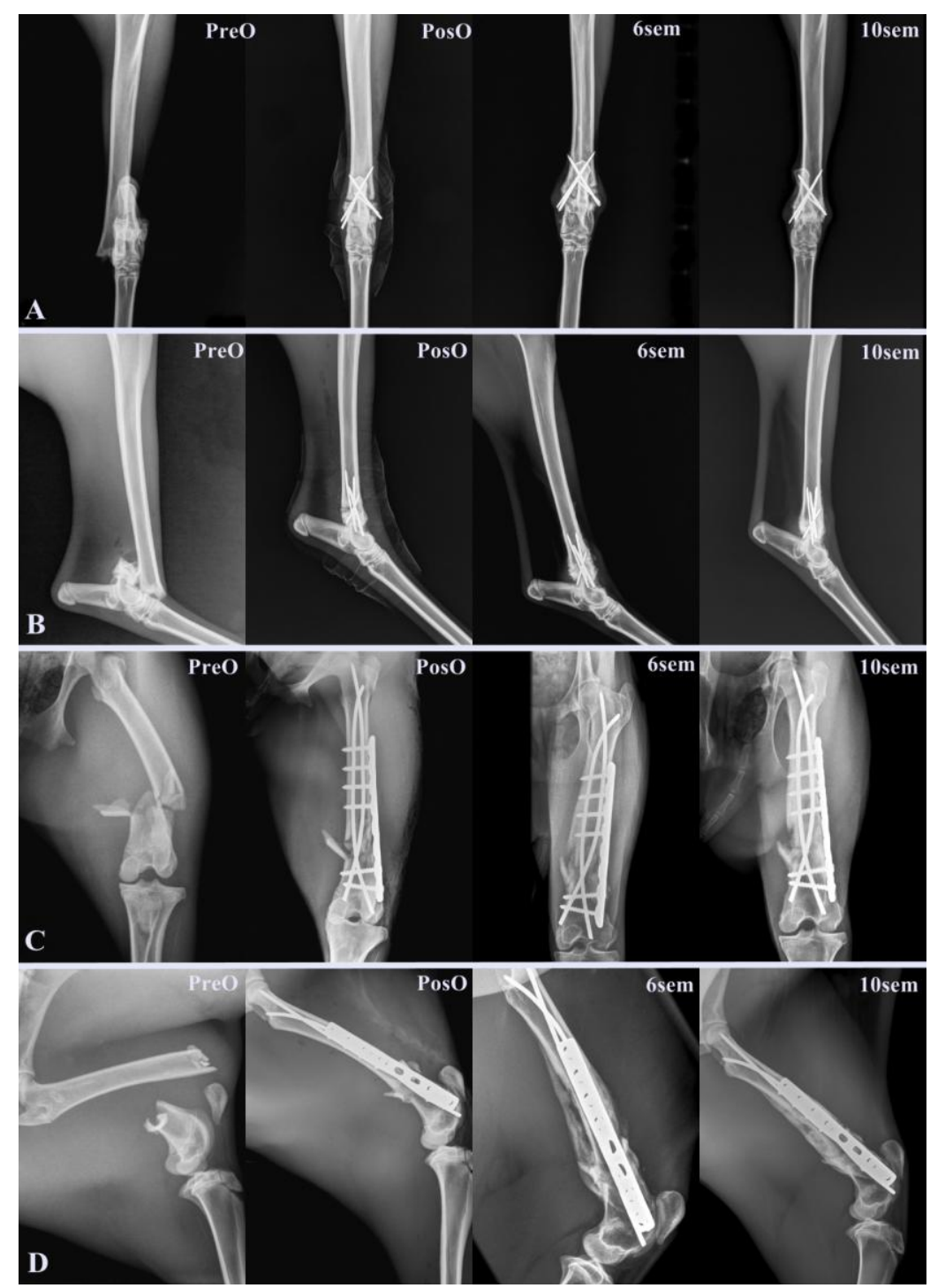

Figura 1. Osteossíntese de fêmur e tíbia em veado-catingueiro (Mazama gouazoubira). A) Imagem radiográfica craniocaudal de tíbia esquerda. B) Imagem radiográfica mediolateral de tíbia esquerda. C) Imagem radiográfica craniocaudal de fêmur esquerdo. D) Imagem radiográfica mediolateral de fêmur esquerdo. PreO: pré-operatório; PosO: pós-operatório imediato; 6sem: seis semanas pós-operatório; 10sem: 10 semanas pós-operatório. 
Exames radiográficos controle foram realizados após seis e 10 semanas das osteossínteses, nos quais foi observada consolidação das fraturas e início da remodelação do calo ósseo (Fig. 1). Após 10 dias das osteossínteses, o animal já apoiava o membro, porém com leve claudicação. Aos 70 dias, já deambulava sem dificuldades e as feridas estavam cicatrizadas e sem indícios de reação local e infecção. Assim, 75 dias após o procedimento cirúrgico, foram removidos três pinos intramedulares da tíbia, mediante anestesia geral, conforme protocolo supracitado.

Após 90 dias do primeiro procedimento cirúrgico, o animal recebeu alta e foi encaminhado para soltura em área determinada pelo órgão ambiental competente.

\section{DISCUSSÃO}

As fraturas distais de fêmur podem ser altamente cominutivas e de difícil redução e estabilização, devido à força da musculatura adjacente e da natureza da fratura, que proporciona pouca disponibilidade óssea distal para acomodação de implantes ortopédicos (Beale, 2004; Harasen, 2002) e significativas forças de flexão (Harasen, 2002). O tratamento cirúrgico tem prognóstico reservado. Fixadores internos são mais recomendados que fixadores externos em virtude da grande quantidade de tecido mole que recobre o osso, podendo causar maior morbidade ao paciente (Beale, 2004). Neste caso, devido à característica cominutiva da fratura e ao comportamento do animal, optou-se pela colocação de pinos intramedulares de Steinmann inseridos conforme a técnica de Rush associado à LC-DCP, visando aumentar a estabilidade e, consequentemente, as chances de sucesso.

Os pinos funcionam durante a consolidação para compartilhar carga e proteger a placa de forças de flexão da sustentação do peso (Piermattei et al., 2009). Em fraturas metafisárias, a reparação com placa pode não permitir parafusos suficientes para serem colocados no fragmento distal. E mesmo quando é possível sua colocação, a extremidade distal da placa pode interferir com fechamento adequado da cápsula articular do joelho e pode afetar a função da parte distal do mecanismo quadríceps-patelar (Harasen, 2002). Como a fratura deste paciente localizava-se na porção mais proximal da metáfise, foi possível a colocação da placa, pois houve espaço para dois parafusos distais ao foco da fratura e não foram observadas complicações referentes à sua colocação.

Em animais jovens é mais frequente a fratura no disco de crescimento, fragilizado pela zona de condrócitos hipertrofiados (Beale, 2004), como ocorreu na fratura distal de tíbia deste paciente. Pinos de Steinmann são comumente utilizados para reparação desse tipo de fratura. A inserção de dois pinos pequenos colocados de maneira diagonal, iniciando nos maléolos medial e lateral, é frequentemente o único método prático de fixação devido ao tamanho reduzido do fragmento. Tal técnica foi realizada, neste caso, com a inserção de quatro pinos para assegurar a estabilidade da redução. Placas não são indicadas devido ao baixo recobrimento de tecido mole sobre essa região do osso e ao pouco espaço para colocação do implante ortopédico (Piermattei et al., 2009).

Na literatura, há relatos de sucesso no tratamento de fraturas cominutivas de rádio, ulna, metacarpo e tíbia com a realização de osteossíntese por meio de técnica fechada, com uso de fixadores esqueléticos externos, em veado-de-cauda-branca (Odocoileus virginianus) e veado-vermelho (Cervus elaphus). Em todos os casos, os autores referem-se à boa aceitação do aparato ortopédico, porém os animais desses relatos eram provenientes de cativeiro (Aitken-Palmer et al., 2012; Phelps et al., 2010, Toews et al., 1998). Nisbet et al. (2010), em um estudo retrospectivo com 20 corças (Capreolus capreolus) que sofreram acidentes traumáticos, referiram-se à ocorrência de fraturas de ossos longos em oito animais. Em apenas três desses foram realizadas osteossínteses, sendo duas delas por técnica aberta, e nenhum deles sobreviveu até cinco dias após a cirurgia. Esse resultado mostra-se diferente do obtido no presente relato, visto que, mesmo o animal apresentando duas fraturas complexas e sendo de vida livre, houve boa evolução clínica e radiográfica.

Tendo em vista o tempo de recuperação do paciente e o retorno de função do membro póscirúrgico, no presente relato, optou-se pela retirada dos pinos intramedulares da tíbia após o completo retorno da funcionalidade locomotora. Entretanto, um dos quatro pinos estava impactado em sua totalidade, e não foi possível sua remoção. Para os implantes do fêmur, em 
razão da vasta cobertura de tecidos moles, da baixa probabilidade de migração e a fim de evitar uma nova intervenção cirúrgica, o que levaria a um maior tempo do animal em cativeiro, optouse pela permanência desses dispositivos. Segundo Catão-Dias e Camargo (2010), períodos prolongados em cativeiro são contraindicados para a espécie devido à possibilidade de ocorrerem novas lesões traumáticas por tentativas de fuga ou óbitos causados por transtornos metabólicos, como miopatia de captura.

Veados selvagens são indóceis, propensos ao estresse e, portanto, de difícil manejo. A captura, contenção física, manutenção e manipulação de cervídeos em cativeiro podem resultar em problemas adicionais e levar a óbito em até 72 horas após o manejo (Catão-Dias e Camargo, 2010). De acordo com Nisbet et al. (2010), a taxa de mortalidade tem relação direta com a não sedação no momento da captura inicial, pois essas medicações são essenciais para redução da resposta ao estresse, minimizando seus efeitos adversos e contribuindo para o sucesso no período de reabilitação pós-operatória. No presente caso, o animal não foi sedado para transporte inicial, pois se apresentava em choque decorrente do trauma, porém, para a avaliação clínica e em todos os procedimentos que pudessem causar alguma agitação, foi utilizado o uso de contenção química, sempre com monitoramento da temperatura corporal e do estado de hidratação do paciente durante todo esse período. Em virtude dessa propensão ao estresse induzido pela manipulação, tratamentos que requerem repetidas aplicações de medicamentos não são recomendados, portanto são preferíveis agentes farmacológicos de ação mais prolongada.

No que se refere ao uso de fármacos em cervídeos, há poucos estudos farmacocinéticos, sendo a grande maioria das doses calculadas por alometria ou extrapoladas do uso em ruminantes domésticos (Nunes e Duarte, 2010). Em relação à analgesia, é importante que ela seja antecipada a procedimentos que possam causar dor (Plummer e Schleining, 2013). Antiinflamatórios não esteroidais são considerados eficazes para dores de baixa a moderada intensidade, proporcionam maior duração da analgesia e meia-vida plasmática mais longa, podendo ser administrados a cada 24 horas. Já o tramadol, assim como alguns opioides, produz níveis consistentes de analgesia, porém com uma meia-vida plasmática que não ultrapassa 2,5 horas, conforme estudo realizado com lhamas (Plummer e Schleining, 2013), podendo ainda, em alguns casos, causar estase e constipação intestinal. Neste caso, optou-se pela realização de bloqueio peridural pré-operatório e pelo uso de meloxicam como analgésico na conduta terapêutica. $O$ tramadol foi utilizado apenas na manipulação inicial, para redução manual da fratura da tíbia e colocação da tala.

O sucesso deste relato pode ser relacionado ao fato de o paciente ser jovem, pois animais assim são menos estressados em comparação aos cervídeos adultos e, portanto, menos propensos a traumatismos em cativeiro, tendo, assim, melhor prognóstico para recuperação (Catão-Dias e Camargo, 2010; Nisbet et al., 2010). Ainda, é evidenciada a importância da sedação do animal para manutenção e manejo dele em cativeiro, ajudando na redução de danos e do tempo de permanência nesse ambiente.

\section{CONCLUSÃO}

Conclui-se que as técnicas cirúrgicas e o manejo clínico adotado foram satisfatórios para estabilização e consolidação das fraturas distais de fêmur e tíbia em veado-catingueiro (Mazama gouazoubira) e favoreceram o adequado retorno da função do membro, propiciando a liberação do animal de volta à natureza. Pelo conhecimento dos autores, este é o primeiro relato de osteossínteses bem-sucedidas de fraturas distais de tíbia e fêmur nesta espécie.

\section{AGRADECIMENTOS}

Os autores agradecem ao Setor de Fauna da Secretaria do Meio Ambiente e Desenvolvimento Sustentável do Estado do Rio Grande do Sul (Sema-RS), em especial ao médico veterinário Elisandro dos Santos, pela assistência no recebimento do animal no PreservasHCV/UFRGS e na logística de destinação/soltura do animal. 


\section{REFERÊNCIAS}

AITKEN-PALMER, C.; LEWIS, D.D.; KIM, S.E.; REESE, D.J. Use of a circular fixator construct for metacarpal fracture stabilization in a fawn. Vet. Rec., v.170, p.444, 2012.

BEALE, B. Orthopedic clinical techniques femur fracture repair. Clin. Tech. Small Anin. Pract., v.19, p.134-150, 2004.

BLACK-DÉCIMA, P.; ROSSI, R.V.; VOGLIOTTI, A.; CARTES, J.L. et al. Brown brocket deer Mazama Gouazoubira (Fisher 1814), In: DUARTE, J.M.B.; GONZÁLEZ, S. (Eds.). Neotropical cervidology: biology and medicine of latin america deer. Jaboticabal: Funep /IUCN, 2010. p.190-201.

CATÃO-DIAS, J.L.; CAMARGO, C.M.S. Capture Myopahy. In: DUARTE, J.M.B.; GONZÁLEZ, S. (Eds.). Neotropical cervidology: biology and medicine of latin america deer. Jaboticabal: Funep /IUCN, 2010. p.324-329.

DUARTE, J.M.B.; REIS, M.L. Plano de ação nacional para conservação dos cervídeos ameaçados de extinção. Brasília: Instituto Chico Mendes de Conservação da Biodversidade, 2012. $126 \mathrm{p}$.

HARASEN, G. Fractures involving the distal extremity of the femur. Part 2: The mature patient. Can. Vet. J., v.43, p.131-132, 2002.
NISBET, H.O.; ÖZAK, A.; YARDIMICI, C.; SIRIN, Y.S. Treatment results of traumatic injuries in 20 Roe Deer (Capreolus capreolus): a retrospective study. Kafkas Univ. Vet. Fak. Derg., v.16, p.617-622, 2010.

NUNES, A.L.V.; DUARTE, J.M.B. Clinical examination and therapeutics. In: DUARTE, J.M.B.; GONZÁLEZ, S. (Eds.). Neotropical cervidology: biology and medicine of latin america deer. Jaboticabal: Funep /IUCN, 2010. p.324-329.

PHELPS, H.A.; LEWIS, D.D., AIKENPALMER, C. Use of a linear-circular hybrid external skeletal fixator for stabilization of a juxta-physeal proximal radial fracture in a deer. J. Zoo Wildl. Med., v.41, p.688-696, 2010.

PIERMATTEI, D.L.; FLO, G.L.; DE CAMP, C.E. Ortopedia e tratamento de fraturas de pequenos animais. 4.ed. Barueri: Manole, 2009. $896 \mathrm{p}$.

PLUMMER, P.J.; SCHLEINING, J.A. Assessment and management of pain in small ruminants and camelids. Vet. Clin. N. Am. Food Am., v.29, p.185-208, 2013.

TOEWS, A.R.; BAILEY, J.V.; THEORET, C. External skeletal fixation for treatment of comminuted fractures in wapiti: 5 cases. Can. Vet. J., v.39, p.370-372, 1998. 